Mathematical Modelling and Analysis

Volume 18 Number 2, April 2013, 176-190

http://dx.doi.org/10.3846/13926292.2013.779943

(c) Vilnius Gediminas Technical University, 2013
Publisher: Taylor\&Francis and VGTU

http://www.tandfonline.com/TMMA

Print ISSN: 1392-6292

Online ISSN: 1648-3510

\title{
On Solvability of Boundary Value Problem for Asymmetric Differential Equation Depending on $x^{\prime}$
}

\section{Armands Gritsans and Felix Sadyrbaev}

Daugavpils University

Parades str. 1, 5400 Daugavpils, Latvia

E-mail(corresp.): armands.gricans@du.lv

E-mail: felix@latnet.lv

Received September 20, 2012; revised January 25, 2013; published online April 1, 2013

\begin{abstract}
We state the conditions of geometrical nature which guarantee the existence of a solution to the boundary value problem $x^{\prime \prime}+2 \delta x^{\prime}+\lambda f\left(x^{+}\right)-\mu g\left(x^{-}\right)=$ $h\left(t, x, x^{\prime}\right), x(0)=0=x(1)$ with a damping term $2 \delta x^{\prime}$ and nonnegative parameters $\lambda$, $\mu$, provided that $f\left(x^{+}\right)-g\left(x^{-}\right)$is a sector-bounded nonlinearity.

Keywords: asymmetric differential equation, Fuč́k type spectrum, comparison, angular functions, Dirichlet boundary value problem.
\end{abstract}

AMS Subject Classification: 34B08; 34B15.

\section{Introduction}

Boundary value problems for asymptotically asymmetric ordinary differential equations attract significant attention in the last decades. This partially can be explained by theoretical reasons: it is natural to study the behaviour of asymmetric oscillators versus symmetric (classical) ones. On the other hand, asymmetric equations appear in applications, such as mechanics. Especially interesting is the study of theory of suspension bridges, since possibly unknown nonlinear phenomena lie in underground of it. The interested reader may consult the works [11] and [12] for additional information and more references.

Let us trace now the development of the theoretical issues connected with the problem under consideration.

The classical harmonic differential equation

$$
x^{\prime \prime}+\lambda x=0
$$

may be changed to the asymmetric equation as follows

$$
x^{\prime \prime}+\lambda x^{+}-\mu x^{-}=0,
$$

where $x^{+}=\max \{x, 0\}, x^{-}=\max \{-x, 0\}$. 
Equation (1.1) when considered together with the boundary conditions

$$
x(0)=0=x(1),
$$

gives rise to eigenvalue problem of finding those values of $\lambda$, for which the problem (1.1), (1.3) has nontrivial solutions (the eigenfunctions). These values of $\lambda$ are called the spectrum of the problem (1.1), (1.3). Knowledge of the spectrum is important when considering the quasi-linear problem

$$
x^{\prime \prime}+\lambda x=h\left(t, x, x^{\prime}\right), \quad x(0)=0=x(1) .
$$

Function $h$ is supposed to be continuous and bounded in modulus. The problem (1.4) is known to be solvable if $\lambda$ does not belong to the spectrum of the problem (1.1), (1.3). In other words, the problem (1.4) has a solution if the homogeneous problem (1.1), (1.3) has only the trivial solution. Otherwise it is resonant problem and it can be solved only for specific nonlinearities $h$.

The similar question about the problem

$$
x^{\prime \prime}+\lambda x^{+}-\mu x^{-}=h\left(t, x, x^{\prime}\right), \quad x(0)=0=x(1)
$$

also can be answered. A set of $(\lambda, \mu)$ such that the problem (1.2), (1.3) has a nontrivial solution is the spectrum of the problem (1.2), (1.3). This spectrum is well known. In the first quadrant of the $(\lambda, \mu)$-plane it consists of two orthogonal straight lines and a set of hyperbolas (this spectrum is called usually the Fučík spectrum). The complement of the spectrum in the first quadrant is a union of two disjoint sets, $S_{1}$ and $S_{2}$. These sets are fully described in the work [8]. It is known [2] that if $(\lambda, \mu) \in S_{1}$ then the problem (1.5) is solvable for bounded nonlinearities $h$.

Nonlinearities can be introduced in equation (1.2) in another way. A nonlinear asymmetric oscillator (in the meaning that restoring forces on the left and right sides nonlinearly depend on replacements $x(t))$ may be associated with equation $x^{\prime \prime}+\lambda f\left(x^{+}\right)-\mu g\left(x^{-}\right)=0$, where $f$ and $g$ are nonlinear nonnegative functions.

In the works $[3,4,5,13]$ the problem

$$
x^{\prime \prime}+\lambda f\left(x^{+}\right)-\mu g\left(x^{-}\right)=0, \quad x(0)=0=x(1)
$$

together with the normalization condition

$$
\left|x^{\prime}(0)\right|=1
$$

was considered. The condition (1.7) is not needed in case of the problem (1.2), (1.3) since it is fulfilled automatically. The spectrum of the problem (1.6), (1.7) is a set of all pairs $(\lambda, \mu)$ such that the problem $(1.6),(1.7)$ has a nontrivial solution - in the first quadrant of the $(\lambda, \mu)$-plane it consists of branches: two orthogonal straight lines and a set of hyperbola looking curves. In [4] some properties of the spectrum were analyzed and was pointed out in particular that branches of the spectrum may have separate components connected at infinity and these separate components can be even bounded. In [5] the problem (1.6), (1.7) was studied provided that the functions $f=g$ are piece-wise linear. 
If we release $\left|x^{\prime}(0)\right|$ from 1 to $\alpha$ the spectrum of the problem (1.6) is a union of solution surfaces [13] - solution surfaces are in the $3 \mathrm{D}$ space $(\lambda, \mu, \alpha)$, where $\alpha=x^{\prime}(0)$. Knowledge of solution surfaces in case of given $f$ and $g$ opens the possibility to evaluate the number of solutions to BVP of the type (1.6). In the works $[6,7]$ the Neumann problem $x^{\prime \prime}+\lambda f\left(x^{+}\right)-\mu g\left(x^{-}\right)=0, x^{\prime}(a)=0=x^{\prime}(b)$, $a<b$, was considered also.

If the problem $x^{\prime \prime}+\lambda f\left(x^{+}\right)-\mu g\left(x^{-}\right)=h\left(t, x, x^{\prime}\right), x(0)=0=x(1)$ is considered with a bounded right side $h$ provided that $f=g$ are sector-bounded nonlinearities [1], the existence results can be obtained also [8]. This can be interpreted as description of an asymmetric oscillator with positive and negative restoring forces given respectively by $f$ and $g=f$ and in presence of external force given by $h$.

In this paper we consider the boundary value problem

$$
x^{\prime \prime}+2 \delta x^{\prime}+\lambda f\left(x^{+}\right)-\mu g\left(x^{-}\right)=h\left(t, x, x^{\prime}\right), \quad x(0)=0=x(1) .
$$

The equation in (1.8) describes an asymmetric oscillator with nonlinear restoring forces $f$ and $g$, an external force $h$ and in presence of damping term given by $2 \delta x^{\prime}$. So despite of asymmetric character of the restoring forces the resistance of medium is symmetric.

\section{Generalization of the classical Fučík spectrum}

In what follows we need to know the spectrum of the boundary value problem

$$
x^{\prime \prime}+2 \delta x^{\prime}+k \lambda x^{+}-k \mu x^{-}=0, \quad x(0)=0=x(1),
$$

where $k>0, \lambda>0, \mu>0$. This problem is a generalization (extension) of the classical Fučík problem [2].

We wish to prove the following result.

Proposition 1. The boundary value problem (2.1) has a nontrivial solution if and only if $(\lambda, \mu)$ is in the Fučik type spectrum $\Sigma_{F}(k, \delta)=\left(\bigcup_{j=0}^{\infty} F_{j}^{+}(k, \delta)\right) \cup$ $\left(\bigcup_{j=0}^{\infty} F_{j}^{-}(k, \delta)\right)$ consisting of the branches

$$
\begin{aligned}
F_{0}^{+}(k, \delta) & =\left\{(\lambda, \mu): \frac{\pi}{\sqrt{k \lambda-\delta^{2}}}=1, \mu \geq 0\right\}, \\
F_{0}^{-}(k, \delta) & =\left\{(\lambda, \mu): \frac{\pi}{\sqrt{k \mu-\delta^{2}}}=1, \lambda \geq 0\right\}, \\
F_{2 i-1}^{+}(k, \delta) & =\left\{(\lambda, \mu): \frac{\pi i}{\sqrt{k \lambda-\delta^{2}}}+\frac{\pi i}{\sqrt{k \mu-\delta^{2}}}=1\right\}, \\
F_{2 i-1}^{-}(k, \delta) & =\left\{(\lambda, \mu): \frac{\pi i}{\sqrt{k \mu-\delta^{2}}}+\frac{\pi i}{\sqrt{k \lambda-\delta^{2}}}=1\right\}, \\
F_{2 i}^{+}(k, \delta) & =\left\{(\lambda, \mu): \frac{\pi(i+1)}{\sqrt{k \lambda-\delta^{2}}}+\frac{\pi i}{\sqrt{k \mu-\delta^{2}}}=1\right\}, \\
F_{2 i}^{-}(k, \delta) & =\left\{(\lambda, \mu): \frac{\pi i}{\sqrt{k \lambda-\delta^{2}}}+\frac{\pi(i+1)}{\sqrt{k \mu-\delta^{2}}}=1\right\},
\end{aligned}
$$

where $i \in \mathbb{N}$. 
Proof. By constructing continuously differentiable solutions with prescribed number of zeros in the interval $(0,1)$.

Suppose for a moment that $k=1$ and consider the problem

$$
x^{\prime \prime}+2 \delta x^{\prime}+\lambda x^{+}-\mu x^{-}=0, \quad x(0)=0=x(1) .
$$

In the case of $\delta^{2}-\lambda<0$ the solution

$$
x(t ; \alpha)=\frac{\alpha}{\sqrt{\lambda-\delta^{2}}} e^{-\delta t} \sin \left(t \sqrt{\lambda-\delta^{2}}\right)
$$

of the Cauchy problem $x^{\prime \prime}+2 \delta x^{\prime}+\lambda x=0, \quad x(0)=0, \quad x^{\prime}(0)=\alpha>0$ has the zeros $t_{i}=i \pi / \sqrt{\lambda-\delta^{2}}(i=1,2, \ldots)$.

"Positive" solutions without zeros. It follows from (2.9) that $x(t ; \alpha)$ is a solution of $(2.8)$ with positive derivative $\left(x^{\prime}(0)>0\right)$ and without zeros in the interval $(0,1)$ if $F_{0}^{+}(1, \delta): \frac{\pi}{\sqrt{\lambda-\delta^{2}}}=1$, where $\lambda-\delta^{2}>0, \mu \geq 0$. If $x^{\prime}(0)=\alpha_{+}>0$, then $x^{\prime}(1)=-\alpha_{+} e^{-\delta}<0$. Therefore the expression for the branch $F_{0}^{+}(1, \delta)$ is obtained.

"Negative" solutions without zeros. Similarly solutions of the problem $(2.8)$ with negative derivative $\left(x^{\prime}(0)<0\right)$ and without zeros in the interval $(0,1)$ arise if $\delta$ and $\mu$ satisfy the relation $F_{0}^{-}(1, \delta): \frac{\pi}{\sqrt{\mu-\delta^{2}}}=1$, where $\mu-\delta^{2}>0$, $\lambda \geq 0$. If $x^{\prime}(0)=\alpha_{-}<0$, then $x^{\prime}(1)=-\alpha_{-} e^{-\delta}>0$. Hence the expression for the branch $F_{0}^{-}(1, \delta)$.

"Positive" solutions with exactly one zero. Consider a solution

$$
x_{\lambda}\left(t ; \alpha_{+}\right)=\frac{\alpha_{+}}{\sqrt{\lambda-\delta^{2}}} e^{-\delta t} \sin \left(t \sqrt{\lambda-\delta^{2}}\right)
$$

of the Cauchy problem $x^{\prime \prime}+2 \delta x^{\prime}+\lambda x=0, x(0)=0, x^{\prime}(0)=\alpha_{+}>0$. The first zero is at $t_{1}^{\lambda}=\frac{\pi}{\sqrt{\lambda-\delta^{2}}}, \lambda-\delta^{2}>0$. Consider also a solution with a negative derivative

$$
x_{\mu}\left(t ; \alpha_{-}\right)=\frac{\alpha_{-}}{\sqrt{\mu-\delta^{2}}} e^{-\delta t} \sin \left(t \sqrt{\mu-\delta^{2}}\right)
$$

of the Cauchy problem $x^{\prime \prime}+2 \delta x^{\prime}+\mu x=0, x(0)=0, x^{\prime}(0)=\alpha_{-}<0$. The first zero is at $t_{1}^{\mu}=\frac{\pi}{\sqrt{\mu-\delta^{2}}}, \mu-\delta^{2}>0$. For a given $\delta$ choose $\lambda$ and $\mu$ such that $F_{1}^{+}(1, \delta): \frac{\pi}{\sqrt{\lambda-\delta^{2}}}+\frac{\pi}{\sqrt{\mu-\delta^{2}}}=1$, where $\lambda-\delta^{2}>0$ and $\mu-\delta^{2}>0$. Both solutions $x_{\lambda}\left(t ; \alpha_{+}\right)$and "shifted" $x_{\mu}\left(t ; \alpha_{-}\right)$combine a solution

$$
x\left(t ; \alpha_{+}\right)= \begin{cases}x_{\lambda}\left(t ; \alpha_{+}\right), & \text {if } 0 \leq t \leq t_{1}^{\lambda}, \\ x_{\mu}\left(t-t_{1}^{\lambda} ; \alpha_{-}\right), & \text {if } t_{1}^{\lambda} \leq t \leq 1\end{cases}
$$

of the problem (2.8) with exactly one zero in the interval $(0,1)$. There is a smooth junction at the first zero $t_{1}^{\lambda}$ if $\alpha_{+}>0, \alpha_{-}=-\alpha_{+} e^{-\delta \frac{\pi}{\sqrt{\lambda-\delta^{2}}}}<0$ and $(\lambda, \mu) \in F_{1}^{+}(1, \delta)$. This solution is positive in the interval $\left(0, t_{1}^{\lambda}\right)$ and negative in $\left(t_{1}^{\lambda}, 1\right)$. 
Summing up, we have that

$$
\begin{aligned}
x\left(0 ; \alpha_{+}\right) & =x_{\lambda}\left(0 ; \alpha_{+}\right)=0, \\
x\left(t_{1}^{\lambda} ; \alpha_{+}\right) & =x_{\lambda}\left(t_{1}^{\lambda} ; \alpha_{+}\right)=x_{\mu}\left(t_{1}^{\lambda}-t_{1}^{\lambda} ; \alpha_{-}\right)=0, \\
x\left(1 ; \alpha_{+}\right) & =x_{\mu}\left(1-t_{1}^{\lambda} ; \alpha_{-}\right)=x_{\mu}\left(t_{1}^{\mu} ; \alpha_{-}\right)=0 ;
\end{aligned}
$$

If $x^{\prime}\left(0 ; \alpha_{+}\right)=\alpha_{+}>0$, then

$$
\begin{gathered}
x^{\prime}\left(t_{1}^{\lambda} ; \alpha_{+}\right)=x_{\lambda}^{\prime}\left(t_{1}^{\lambda} ; \alpha_{+}\right)=-\alpha_{+} e^{-\delta \frac{\pi}{\sqrt{\lambda-\delta^{2}}}}=\alpha_{-}<0 \\
x^{\prime}\left(t_{1}^{\lambda} ; \alpha_{+}\right)=x_{\mu}^{\prime}\left(t_{1}^{\lambda}-t_{1}^{\lambda} ; \alpha_{-}\right)=x_{\mu}^{\prime}\left(0 ; \alpha_{-}\right)=\alpha_{-}<0 \\
x^{\prime}\left(1 ; \alpha_{+}\right)=x_{\mu}^{\prime}\left(1-t_{1}^{\lambda} ; \alpha_{-}\right)=x_{\mu}^{\prime}\left(t_{1}^{\mu} ; \alpha_{-}\right)=\alpha_{+} e^{-\delta} .
\end{gathered}
$$

"Negative" solutions with exactly one zero. Similarly the case of a solution with $x^{\prime}(0)<0$, which has exactly one zero in $(0,1)$, can be considered. For a given $\delta$ choose $\lambda$ and $\mu$ such that $F_{1}^{-}(1, \delta): \frac{\pi}{\sqrt{\mu-\delta^{2}}}+\frac{\pi}{\sqrt{\lambda-\delta^{2}}}=1$, where $\lambda-\delta^{2}>0$ and $\mu-\delta^{2}>0$. The desired solution of the problem (2.8) is obtained combining the solution $x_{\mu}\left(t ; \alpha_{-}\right)$and "shifted" solution $x_{\lambda}\left(t ; \alpha_{+}\right)$as

$$
x\left(t ; \alpha_{-}\right)= \begin{cases}x_{\mu}\left(t ; \alpha_{-}\right), & \text {if } 0 \leq t \leq t_{1}^{\mu}, \\ x_{\lambda}\left(t-t_{1}^{\mu} ; \alpha_{+}\right), & \text {if } t_{1}^{\mu} \leq t \leq 1\end{cases}
$$

The junction at the first zero point $t_{1}^{\mu}$ is smooth if $\alpha_{-}<0, \alpha_{+}=-\alpha_{-}$ $e^{-\delta \frac{\pi}{\sqrt{\mu-\delta^{2}}}}>0$ and $(\lambda, \mu) \in F_{1}^{-}(1, \delta)$. The obtained solution is negative in the interval $\left(0, t_{1}^{\mu}\right)$ and positive in $\left(t_{1}^{\mu}, 1\right)$. Hence the expression for the branch $F_{1}^{-}(1, \delta)$.

Replacing $\lambda$ and $\mu$ in the above considerations by $k \lambda$ and $k \mu$ respectively we obtain the expressions (2.2)-(2.7) for the branches of the spectrum of the problem (2.1).

Remark 1. 1) A branch $F_{j}^{s}(k, \delta)$ describes all nontrivial "positive" (resp.: "negative") solutions of the problem (2.1), that is, all nontrivial solutions with $x^{\prime}(0)>0$ (resp.: $x^{\prime}(0)<0$ ) of the problem $(2.1)$, with $j$ zeroes in the interval $(0,1)$ if $s=+(s=-)$;

2) If $x(t)$ is a nontrivial solution of the problem (2.1), then the derivatives $x^{\prime}(z)$ at $j$-th zeros $z \in[0,1]$ of the solution $x(t)$ (from left to right) are:

- for a "positive" solution

$$
\begin{gathered}
x^{\prime}(0)=\alpha>0,-\alpha e^{-\delta \frac{\pi}{\sqrt{k \lambda-\delta^{2}}}>0, \alpha e^{-\delta\left(\frac{\pi}{\sqrt{k \lambda-\delta^{2}}}+\frac{\pi}{\sqrt{k \mu-\delta^{2}}}\right)},} \\
-\alpha e^{-\delta\left(\frac{2 \pi}{\sqrt{k \lambda-\delta^{2}}}+\frac{\pi}{\sqrt{k \mu-\delta^{2}}}\right)}, \alpha e^{-\delta\left(\frac{2 \pi}{\sqrt{k \lambda-\delta^{2}}}+\frac{2 \pi}{\sqrt{k \mu-\delta^{2}}}\right)}, \ldots, x^{\prime}(1)=(-1)^{j+1} \alpha e^{-\delta},
\end{gathered}
$$

- for a "negative" solution

$$
\begin{gathered}
x^{\prime}(0)=\alpha<0,-\alpha e^{-\delta \frac{\pi}{\sqrt{k \mu-\delta^{2}}}}>0, \alpha e^{-\delta\left(\frac{\pi}{\sqrt{k \mu-\delta^{2}}}+\frac{\pi}{\sqrt{k \lambda-\delta^{2}}}\right)}, \\
-\alpha e^{-\delta\left(\frac{2 \pi}{\sqrt{k \mu-\delta^{2}}}+\frac{\pi}{\sqrt{k \lambda-\delta^{2}}}\right)}, \alpha e^{-\delta\left(\frac{2 \pi}{\sqrt{k \mu-\delta^{2}}}+\frac{2 \pi}{\sqrt{k \lambda-\delta^{2}}}\right)}, \ldots, x^{\prime}(1)=(-1)^{j+1} \alpha e^{-\delta} .
\end{gathered}
$$




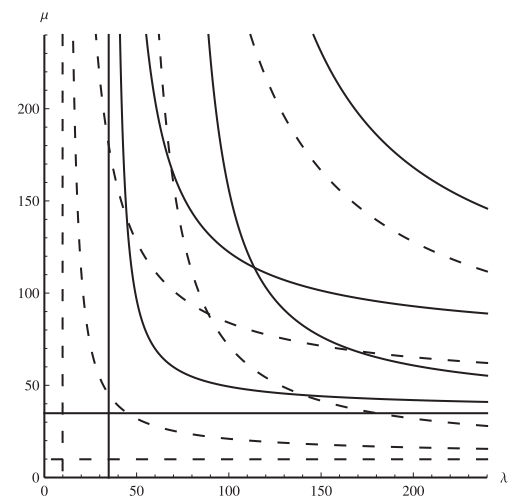

Figure 1. The Fučík type spectrum $\Sigma_{F}(1,5)$ (solid) can be obtained from the classical Fučík spectrum $\Sigma_{F}(1,0)$ (dashed) by the translation parallel to the vector $\left(5^{2}, 5^{2}\right)$.

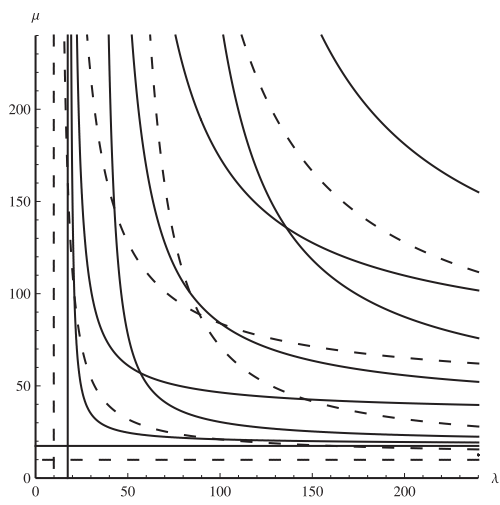

Figure 2. The Fučík type spectrum $\Sigma_{F}(2,5)$ (solid) can be obtained from the classical Fučík spectrum $\Sigma_{F}(1,0)$ (dashed) by composition of translation parallel to the vector $\left(5^{2}, 5^{2}\right)$ and homothety with the center $(0,0)$ and coefficient $\frac{1}{2}$.

3) The branches of the Fučík type spectrum $\Sigma_{F}(k, \delta)$ can be obtained from the classical Fučík spectrum $\Sigma_{F}(1,0)$ by composition of the translation parallel to the vector $\left(\delta^{2}, \delta^{2}\right)$ and the homothety with the center $(0,0)$ and coefficient $\frac{1}{k}$, hence the branches $F_{j}^{s}(k, \delta)(j>0)$ are located in the sector $\{(\lambda, \mu): \lambda>$ $\left.\frac{\delta^{2}}{k}, \mu>\frac{\delta^{2}}{k}\right\}$; see Figs. 1 and 2 .

\section{Solvability of the problem (3.1)}

We wish to prove the existence results for the boundary value problem

$$
x^{\prime \prime}+2 \delta x^{\prime}+\lambda f\left(x^{+}\right)-\mu g\left(x^{-}\right)=h\left(t, x, x^{\prime}\right), \quad x(0)=0=x(1) .
$$

where $f$ and $g$ are sector-bounded nonlinearities and the damping term $2 \delta x^{\prime}$ is present. The left-hand side is called a principal part of the problem (3.1). We are looking for (sufficient) conditions on the parameters $\lambda, \mu>0$ and the damping coefficient $\delta$ which guarantee the existence of a solution to the problem (3.1).

We suppose that the following conditions are fulfilled.

A) $f, g:[0,+\infty) \rightarrow[0,+\infty)$ are locally Lipshitz functions and $f(0)=g(0)=$ 0

B) sector-bounded nonlinearity condition: $l x<f(x)<k x, l x<g(x)<k x$ for $x>0$, where $0<l<k$; then $x\left(\lambda l x^{+}-\mu l x^{-}\right)<x\left(\lambda f\left(x^{+}\right)-\mu g\left(x^{-}\right)\right)<$ $x\left(\lambda k x^{+}-\mu k x^{-}\right)$for $x \neq 0, \lambda, \mu>0$;

C) $h:[0,1] \times \mathbb{R} \times \mathbb{R} \rightarrow \mathbb{R}$ is continuous bounded locally Lipshitz (with respect to $x$ and $x^{\prime}$ ) function. 


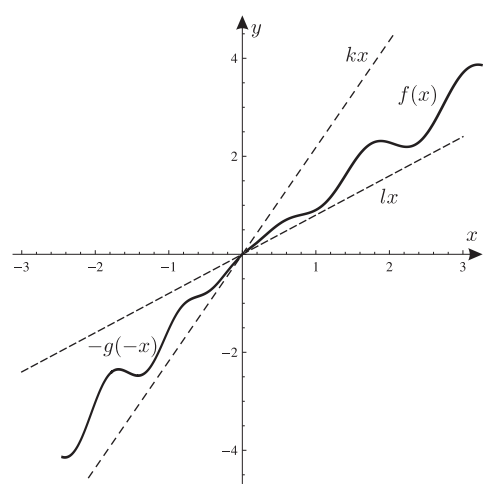

Figure 3. Visualization of sector-bounded nonlinearity $f\left(x^{+}\right)-g\left(x^{-}\right)$.

Remark 2. 1) The sector-bounded nonlinearity concept, see Fig. 3, was used in Aizerman's conjecture [1] and exploited in the feedback systems theory [9].

2) Roughly speaking, the problem (3.1) has a solution if a triple $(k, l, \delta)$ satisfies some requirements. In order to make this point precise, we introduce the sets $D_{j}(k, \delta)$.

\subsection{Regions $D_{j}(k, \delta)$}

Suppose that $k>0$ and consider the problem (2.1) with the spectrum $\Sigma_{F}(k, \delta)$ described in Proposition 1.

Let us introduce a subset $D_{j}(k, \delta)(j=0,1,2, \ldots)$ of the first quadrant in the $(\lambda, \mu)$-plane in which the solutions of the initial value problems

$$
x^{\prime \prime}+2 \delta x^{\prime}+k \lambda x^{+}-k \mu x^{-}=0, \quad x(0)=0, \quad x^{\prime}(0)= \pm 1
$$

have opposite signs at $t=1$ and exactly $j$ zeros in $(0,1)$.

Proposition 2. Suppose $k>0$. The regions $D_{j}(k, \delta)(j=0,1,2, \ldots)$ are

$$
\begin{aligned}
D_{0}(k, \delta)= & \left.(\lambda, \mu): \frac{\pi}{\sqrt{k \lambda-\delta^{2}}}>1, \frac{\pi}{\sqrt{k \mu-\delta^{2}}}>1\right\} ; \\
D_{j}(k, \delta)= & \left\{(\lambda, \mu): \frac{\pi(i-1)}{\sqrt{k \lambda-\delta^{2}}}+\frac{\pi i}{\sqrt{k \mu-\delta^{2}}}<1, \frac{\pi i}{\sqrt{k \lambda-\delta^{2}}}+\frac{\pi(i-1)}{\sqrt{k \mu-\delta^{2}}}<1,\right. \\
& \left.\frac{\pi i}{\sqrt{k \lambda-\delta^{2}}}+\frac{\pi i}{\sqrt{k \mu-\delta^{2}}}>1\right\} \quad \text { if } j=2 i-1(i \in \mathbb{N}) ; \\
D_{j}(k, \delta)= & \left\{(\lambda, \mu): \frac{\pi i}{\sqrt{k \lambda-\delta^{2}}}+\frac{\pi(i+1)}{\sqrt{k \mu-\delta^{2}}}>1, \frac{\pi(i+1)}{\sqrt{k \lambda-\delta^{2}}}+\frac{\pi i}{\sqrt{k \mu-\delta^{2}}}>1,\right. \\
& \left.\frac{\pi i}{\sqrt{k \lambda-\delta^{2}}}+\frac{\pi i}{\sqrt{k \mu-\delta^{2}}}<1\right\} \quad \text { if } j=2 i(i \in \mathbb{N}),
\end{aligned}
$$

where $k \lambda-\delta^{2}>0$ and $k \mu-\delta^{2}>0$. 


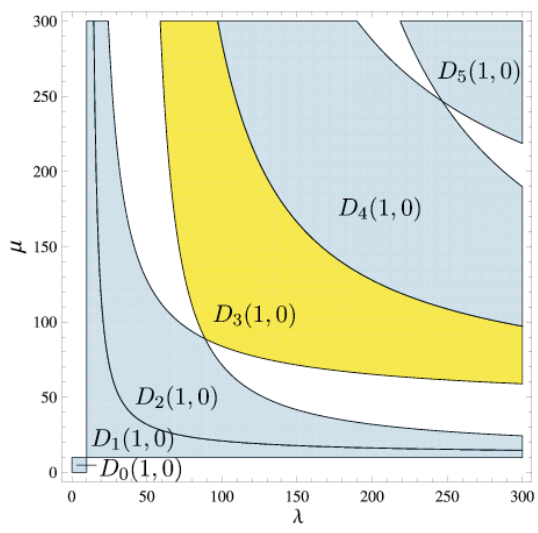

Figure 4. "Good" regions $D_{j}(1,0)$ $(j=0,1, \ldots, 5)$ shaded.

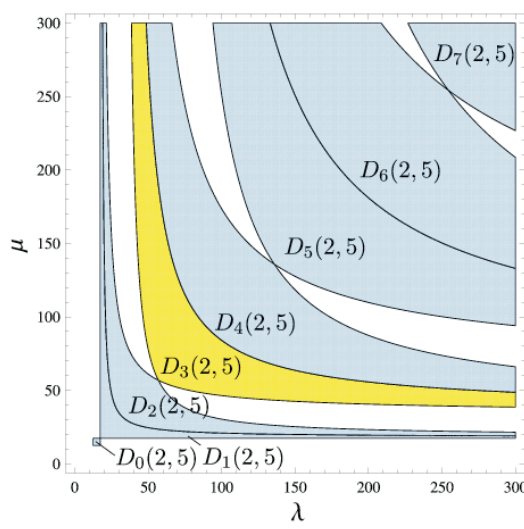

Figure 5. "Good" regions $D_{j}(2,5)$ $(j=0,1, \ldots, 7)$ shaded.

Proof is obtained by direct calculations.

Remark 3. From Remark 1 follows that a region $D_{j}(k, \delta)$, where $j \in\{0,1,2, \ldots\}$ and $k>0$, can be obtained from the region $D_{j}(1,0)$ by composition of translation parallel to the vector $\left(\delta^{2}, \delta^{2}\right)$ and homothety with the center $(0,0)$ and coefficient $\frac{1}{k}$. Besides all regions $D_{j}(k, \delta)$ are located in the sector $\{(\lambda, \mu): \lambda>$ $\left.\frac{\delta^{2}}{k}, \mu>\frac{\delta^{2}}{k}\right\}$. In Figs. 4 and 5 some first regions $D_{j}(1,0)$ and $D_{j}(2,5)$ are depicted. One can see how the region $D_{3}(1,0)$ transforms to the region $D_{3}(2,5)$ (both regions in yellow) under the above mentioned composition. It is worth to note that all regions $D_{j}(k, \delta)(j=0,1,2, \ldots)$ are open connected sets; bounded if $j=0$ and unbounded if $j>0$; two regions $D_{j_{1}}(k, \delta)$ and $D_{j_{2}}(k, \delta)$, where $j_{1}, j_{2} \in\{0,1,2, \ldots\}$, do not intersect if $j_{1} \neq j_{2}$.

\subsection{Some preparations to Main Theorem}

Since it is essential for proving the main result, we discuss solvability of the problem (3.1) when $f(x)=g(x)=x$ for all $x \geq 0$ and $h$ satisfies the condition $\mathbf{C})$. Respectively, consider the problem

$$
x^{\prime \prime}+2 \delta x^{\prime}+\lambda x^{+}-\mu x^{-}=h\left(t, x, x^{\prime}\right), \quad x(0)=0, \quad x(1)=0 .
$$

It follows from Proposition 1 that if $(\lambda, \mu) \notin \Sigma_{F}(1, \delta)$ then the problem (2.1) with $k=1$ has only the trivial solution. This is insufficient for solvability of the problem (3.6). The solvability, however, can be guaranteed for regions $D_{j}(1, \delta)(j=0,1,2, \ldots)$ - "good" regions for the problem $(3.6)$.

Consider the Cauchy problem

$$
x^{\prime \prime}+2 \delta x^{\prime}+\lambda x^{+}-\mu x^{-}=h\left(t, x, x^{\prime}\right), \quad x(0)=0, \quad x^{\prime}(0)=\alpha .
$$

Introduce the functions $u(t)$ and $v(t)$ as solutions of the Cauchy problems

$$
u^{\prime \prime}+2 \delta u^{\prime}+\lambda u^{+}-\mu u^{-}=0, \quad u(0)=0, \quad u^{\prime}(0)=1
$$


and

$$
v^{\prime \prime}+2 \delta v^{\prime}+\lambda v^{+}-\mu v^{-}=0, \quad v(0)=0, \quad v^{\prime}(0)=-1 .
$$

Let $x(t ; \alpha)$ be a solution of (3.7). The normalized functions $y(t ; \alpha)=x(t ; \alpha) / \alpha$ tend respectively to the functions $u(t)$ and $v(t)$ as $\alpha \rightarrow \pm \infty$. Notice that $y(t ; \alpha)$ satisfies also the equation

$$
y^{\prime \prime}+2 \delta y^{\prime}+\lambda y^{+}-\mu y^{-}=\frac{1}{\alpha} h\left(t, x, x^{\prime}\right),
$$

where $\frac{1}{\alpha} h\left(t, x, x^{\prime}\right)$ tends to zero uniformly in $t, x, x^{\prime}$ as $\alpha \rightarrow \infty$. If

$$
y(1 ;+\infty) y(1 ;-\infty)<0
$$

or, which is equivalent,

$$
u(1) v(1)<0,
$$

then the existence of $x\left(t ; \alpha_{0}\right)$ which solves the problem (3.6) can be concluded.

Therefore the problem (3.6) is solvable if (3.8) holds, that is, $(\lambda, \mu)$ is in a "good" region $D_{j}(1, \delta)$, see $(3.3)-(3.5)$, for a some $j \in\{0,1,2, \ldots\}$.

\subsection{The Main Theorem: statement}

The next theorem states the main result.

Theorem 1 [Main Theorem]. Suppose that functions $f, g$ and $h$ satisfy the conditions $\mathbf{A}), \mathbf{B})$ and $\mathbf{C})$. If $(\lambda, \mu)$ is in the region $D_{j}(k, l, \delta)=D_{j}(k, \delta) \cap$ $D_{j}(l, \delta)$ for some $j \in\{0,1,2, \ldots\}$, then the problem (3.1) has a solution.

One can say for brevity that nonempty regions $D_{j}(k, l, \delta)(j=0,1,2, \ldots)$ are "good" regions for the problem (3.1).

To prove the Main Theorem, we need some comparison results.

\subsection{Auxiliary results on angular functions}

\subsubsection{Differential inequality}

The below arguments almost one-by-one repeat the analogous considerations in the paper of the authors [8].

The following assertion is a slight modification of Theorem 14.1 in [10].

Theorem 2. Let $\varphi(t)$ and $\psi(t)$ be $C^{1}([a, b])$ functions which satisfy

$$
\frac{d \varphi}{d t}>F(t, \varphi(t)), \quad \frac{d \psi}{d t}=F(t, \psi(t)), \quad a \leq t \leq b
$$

and $\varphi(a)=\psi(a)$, where $F \in C([a, b], R)$. Then $\varphi(t)>\psi(t)$ for $a<t \leq b$. If $\frac{d \varphi}{d t}<F(t, \varphi(t))$ and $\varphi(a)=\psi(a)$, then $\varphi(t)<\psi(t)$ for $a<t \leq b$.

Proof. Evidently $\frac{d \varphi}{d t}(a)>\frac{d \psi}{d t}(a)$. Therefore $\varphi(t)>\psi(t)$ for $t \in(a, a+\varepsilon)$ for some positive $\varepsilon$. The graph of $\varphi(t)$ cannot cross the graph of $\psi(t)$ downwards. Therefore $\varphi(t)>\psi(t)$ for $t \in(a, b]$. 


\subsubsection{Angular functions}

In what follows we restate the comparison results of [10, Ch. 15] adapted for the case under consideration.

Consider two the second order equations written in a form of two-dimensional systems

$$
\left\{\frac{d x}{d t}=y, \quad \frac{d y}{d t}=-q(x)\right.
$$

and

$$
\left\{\frac{d x}{d t}=y, \quad \frac{d y}{d t}=-\tilde{q}(x) .\right.
$$

Suppose that

$$
x q(x)>x \tilde{q}(x), \quad x \neq 0
$$

and $\tilde{q}(c x)=c \tilde{q}(x)$ for $c>0$. The latter means that function $\tilde{q}(x)$ is positive homogeneous.

Introduce the polar coordinates as $x(t)=r(t) \sin \varphi(t), x^{\prime}(t)=r(t) \cos \varphi(t)$ and let $(r(t), \varphi(t))$ and $(\tilde{r}(t), \tilde{\varphi}(t))$ be coordinates for (3.9) and (3.10) respectively. One gets for $\varphi(t)$ and $\tilde{\varphi}(t)$ that

$$
\frac{d \varphi}{d t}=\frac{1}{r}\left[r \cos ^{2} \varphi+q(r \sin \varphi) \sin \varphi\right] .
$$

On the other hand,

$$
\begin{aligned}
\frac{d \tilde{\varphi}}{d t} & =\frac{1}{\tilde{r}}\left[\tilde{r} \cos ^{2} \tilde{\varphi}+\tilde{q}(\tilde{r} \sin \tilde{\varphi}) \sin \tilde{\varphi}\right] \\
& =\cos ^{2} \tilde{\varphi}+\tilde{q}(\sin \tilde{\varphi}) \sin \tilde{\varphi}:=F(\tilde{\varphi}) .
\end{aligned}
$$

It follows from $(3.11)$ that $q(r \sin \varphi) \sin \varphi>\tilde{q}(r \sin \varphi) \sin \varphi$ if $\varphi \neq 0(\bmod \pi)$ and therefore $\frac{d \varphi(t)}{d t}>F(\varphi(t))$ and, if $\varphi(a)=\tilde{\varphi}(a)$, then, by Theorem $2, \varphi(t)>\tilde{\varphi}(t)$ for any $t \in(a, b]$.

If inequality (3.11) is changed to the opposite then $\frac{d \varphi(t)}{d t}<F(\varphi(t))$ and, if $\varphi(a)=\tilde{\varphi}(a)$, then, by Theorem $2, \varphi(t)<\tilde{\varphi}(t)$ for any $t \in(a, b]$.

\subsubsection{Comparison of angular functions}

Consider shortened equation

$$
x^{\prime \prime}=-2 \delta x^{\prime}-\lambda f\left(x^{+}\right)+\mu g\left(x^{-}\right)
$$

and compare it to equations

$$
\begin{aligned}
& x^{\prime \prime}=-2 \delta x^{\prime}-k \lambda x^{+}+k \mu x^{-}, \\
& x^{\prime \prime}=-2 \delta x^{\prime}-l \lambda x^{+}+l \mu x^{-}
\end{aligned}
$$

having in mind the conditions $\mathbf{B}$ ).

Notice that

$$
\begin{aligned}
x\left(2 \delta x^{\prime}+\lambda k x^{+}-\mu k x^{-}\right) & >x\left(2 \delta x^{\prime}+\lambda f\left(x^{+}\right)-\mu g\left(x^{-}\right)\right) \\
& >x\left(2 \delta x^{\prime}+\lambda l x^{+}-\mu l x^{-}\right), \quad x \neq 0 .
\end{aligned}
$$


The right sides of equations (3.14) and (3.15) are positive homogeneous functions, therefore the arguments of preceding subsection are applicable.

If $\varphi_{k}(t), \varphi(t)$ and $\varphi_{l}(t)$ are the angular functions for equations (3.14), (3.13), (3.15) respectively, one has that

$$
\varphi_{k}(t)>\varphi(t)>\varphi_{l}(t), \quad t \in(0,1]
$$

if $\varphi_{k}(0)=\varphi(0)=\varphi_{l}(0)$.

Thus we have arrived to the following result.

Lemma 1. Let $(\lambda, \mu)$ be in $D_{j}(k, l, \delta)=D_{j}(k, \delta) \cap D_{j}(l, \delta)$ for some $j \in$ $\{0,1,2, \ldots\}$. Then the angular functions for equations (3.14), (3.13), (3.15), which satisfy $\varphi_{k}(0)=\varphi(0)=\varphi_{l}(0)=\varphi_{0}, \varphi_{0}=0$ or $\varphi_{0}=\pi$ satisfy also the inequalities (3.17).

Remark 4. The above lemma means that for $(\lambda, \mu) \in D_{j}(k, l, \delta)$ any solution of equation (3.13) with the initial conditions $x(0)=0, x^{\prime}(0)>0$ has exactly $j$ zeros in $(0,1)$ and $x(1) \neq 0$. The same is true for solutions of equation (3.13) with the initial conditions $x(0)=0, x^{\prime}(0)<0$.

\subsection{Proof of Main Theorem}

Consider equation

$$
x^{\prime \prime}=-2 \delta x^{\prime}-\lambda f\left(x^{+}\right)+\mu g\left(x^{-}\right)+h\left(t, x, x^{\prime}\right) .
$$

Due to the condition B) functions $f$ and $g$ can be represented as

$$
f(x)=l x+\kappa(x)(k-l) x, \quad g(x)=l x+\chi(x)(k-l) x
$$

for $x>0$, where $0<\kappa(x)<1,0<\chi(x)<1$ for $x>0$. This allows to rewrite equation (3.18) as

$x^{\prime \prime}+2 \delta x^{\prime}+\lambda\left[l x^{+}+\kappa\left(x^{+}\right)(k-l) x^{+}\right]-\mu\left[l x^{-}+\chi\left(x^{-}\right)(k-l) x^{-}\right]=h\left(t, x, x^{\prime}\right)$.

Consider the Cauchy problem

$$
x(0)=0, \quad x^{\prime}(0)=\gamma
$$

for equation (3.20), where $\gamma$ will tend to $+\infty$. The functions $u(t ; \gamma):=\frac{1}{\gamma} x(t ; \gamma)$ satisfy

$$
\begin{aligned}
u^{\prime \prime} & +2 \delta u^{\prime}+\lambda\left[l u^{+}+\kappa\left(\gamma u^{+}\right)(k-l) u^{+}\right]-\mu\left[l u^{-}+\chi\left(\gamma u^{-}\right)(k-l) u^{-}\right] \\
& =\frac{1}{\gamma} h\left(t, \gamma u, \gamma u^{\prime}\right)
\end{aligned}
$$

$$
u(0)=0, \quad u^{\prime}(0)=1 .
$$

The right side in (3.22) tends to zero as $\gamma \rightarrow+\infty$ uniformly in $t$. Functions $\kappa$ and $\chi$ take values between 0 and 1 for all $\gamma$ and $t \in[0,1]$. Therefore functions 
$u(t ; \gamma)$ behave similarly (in particular, have the same number of zeros in the interval $(0,1])$ to functions $v(t ; \gamma)$, which solve the problem

$$
\begin{aligned}
& v^{\prime \prime}+2 \delta v^{\prime}+\lambda\left[l v^{+}+\kappa\left(\gamma v^{+}\right)(k-l) v^{+}\right]-\mu\left[l v^{-}+\chi\left(\gamma v^{-}\right)(k-l) v^{-}\right]=0, \\
& v(0)=0, \quad v^{\prime}(0)=1
\end{aligned}
$$

Functions $v(t ; \gamma)$ are equal, in turn, to functions $\frac{1}{\gamma} y(t ; \gamma)$, where $y(t ; \gamma)$ solve the problems

$$
\begin{aligned}
& y^{\prime \prime}+2 \delta y^{\prime}+\lambda\left[l y^{+}+\kappa\left(y^{+}\right)(k-l) y^{+}\right]-\mu\left[l y^{-}+\chi\left(y^{-}\right)(k-l) y^{-}\right]=0, \\
& y(0)=0, \quad y^{\prime}(0)=\gamma
\end{aligned}
$$

Equation (3.25) actually is

$$
y^{\prime \prime}=-2 \delta y^{\prime}-\lambda f\left(y^{+}\right)+\mu g\left(y^{-}\right) .
$$

By Lemma 1, solutions $y(t ; \gamma)$ with the initial conditions $y(0)=0, y^{\prime}(0)=\gamma>$ 0 have exactly $j$ zeros in $(0,1)$ and $y(1 ; \gamma) \neq 0$. The same is true for solutions $x(t ; \gamma)$ of the problem (3.18), (3.21), when $\gamma \rightarrow+\infty$.

The above arguments can be repeated for $\gamma \rightarrow-\infty$. The respective solutions $x(t ; \gamma)$ of the problem $(3.18),(3.21)$ have exactly $j$ zeros in $(0,1)$ and $x(1 ; \gamma) \neq 0$ for $\gamma$ negative and large enough in modulus. Since $x^{\prime}(0 ; \gamma)$ are of opposite sign for $\gamma \rightarrow \pm \infty$, the values $x(1 ; \gamma)$ are also of opposite signs for $\gamma \rightarrow \pm \infty$. By continuity arguments there exists $\gamma_{0}$ such that $x\left(1 ; \gamma_{0}\right)=0$. Then $x\left(t ; \gamma_{0}\right)$ solves the problem (1.8). Hence the proof.

Remark 5. An example can be constructed following the below instructions. Suppose $k>l>0$ are given numbers. Take $f, g \in C^{1}[0,+\infty)$ such that $l<\frac{f(x)}{x}<k$ and $l<\frac{g(x)}{x}<k$ for $x>0$. Then $f(0)=g(0)=0$. Consider equation

$$
x^{\prime \prime}+2 \delta x^{\prime}+\lambda f\left(x^{+}\right)-\mu g\left(x^{-}\right)=h\left(t, x, x^{\prime}\right),
$$

where $h$ is any function which satisfies the condition $\mathbf{C})$. Then a solution $x(t)$ of the problem (1.8) exists for $(\lambda, \mu) \in D_{j}(k, l, \delta)$.

\section{Remarks on regions $D_{i}(k, l, \delta)$}

Proposition 3. Suppose $0<l<k$. A region $D_{j}(k, l, \delta)=D_{j}(k, \delta) \cap D_{j}(l, \delta)$, where $j \in\{0,1,2, \ldots\}$, is nonempty if and only if the damping coefficient $\delta$ and the $(l, k)$-sector satisfy the conditions

$$
\frac{k}{l}<\left(\frac{j+1}{j}\right)^{2} \quad \text { and } \quad|\delta|<\pi \sqrt{\frac{(j+1)^{2} l-j^{2} k}{k-l}} .
$$

Proof. First notice that the branch $F_{j}^{s}(k, \delta)$ of the spectrum $\sum_{F}(k, \delta)$, where $j \in\{0,1,2, \ldots\}$ and $s=+$ or $s=-$, crosses the bisectrix $\lambda=\mu$ at the point $A_{j}(k, \delta)=\left(\frac{\pi^{2}(j+1)^{2}+\delta^{2}}{k}, \frac{\pi^{2}(j+1)^{2}+\delta^{2}}{k}\right)$. Introduce natural ordering on the bisectrix $\lambda=\mu$ for $\lambda>0$. We write $A \prec B$ if $A$ precedes $B$ on the bisectrix. It 
is convenient to denote also $A_{-1}(k, \delta)=\left(\frac{\delta^{2}}{k}, \frac{\delta^{2}}{k}\right)$. Then $A_{-1}(k, \delta) \prec A_{0}(k, \delta) \prec$ $A_{1}(k, \delta) \prec \cdots \prec A_{j}(k, \delta) \prec \cdots$. The borders of the region $D_{j}(k, \delta)$ crosses the bisectrix $\lambda=\mu$ at the points $A_{j-1}(k, \delta)$ and $A_{j}(k, \delta)$. We will call these points the lower diagonal point of the region $D_{j}(k, \delta)$ and upper diagonal point.

It follows from $0<l<k$ that $0<\frac{1}{k}<\frac{1}{l}$. Therefore $A_{j-1}(k, \delta) \prec$ $A_{j-1}(l, \delta) \prec A_{j}(l, \delta)$, which implies that the condition $D_{j}(k, l, \delta)=D_{j}(k, \delta) \cap$ $D_{j}(l, \delta) \neq \varnothing$ is equivalent to the condition that the upper diagonal point of $D_{j}(k, \delta)$ is greater (in the sense $\succ$ ) than the lower diagonal point of $D_{j}(l, \delta)$, that is $A_{j}(k, \delta) \succ A_{j-1}(l, \delta)$, or $\delta^{2}<\pi^{2} \frac{(j+1)^{2} l-j^{2} k}{k-l}$. If $\frac{k}{l}<\left(\frac{j+1}{j}\right)^{2}$, then the second inequality of (4.1) fulfils.

Hence the alternative form of the main theorem can be given.

Theorem 3. Let functions $f, g$ and $h$ satisfy the conditions $\mathbf{A}), \mathbf{B})$ and $\mathbf{C})$. If there exists $j \in\{0,1,2, \ldots\}$ such that the damping coefficient $\delta$ and the $(l, k)$ sector satisfy the conditions (4.1), then there exists a pair of positive parameters $(\lambda, \mu)$ in the region $D_{j}(k, l, \delta)=D_{j}(k, \delta) \cap D_{j}(l, \delta)$ such that the problem $(3.1)$ has a solution.

Let us give the geometrical interpretation of the conditions (4.1), considering the specific $(l, k)$-sector, where $k>1$ and $l=\frac{1}{k}<1$. The sector is symmetric with respect to the bisectrix $\lambda=\mu$. In this case $D_{j}\left(k, \frac{1}{k}, \delta\right) \neq \varnothing$ iff

$$
k<\frac{j+1}{j} \text { and }|\delta|<\pi \sqrt{\frac{(j+1)^{2}-j^{2} k^{2}}{k^{2}-1}} .
$$

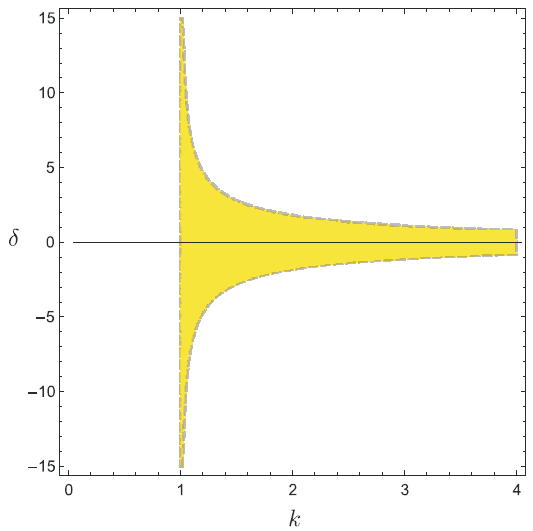

Figure 6. $D_{0}\left(k, \frac{1}{k}, \delta\right)$ is nonempty iff $(k, \delta)$ is in the shaded region.

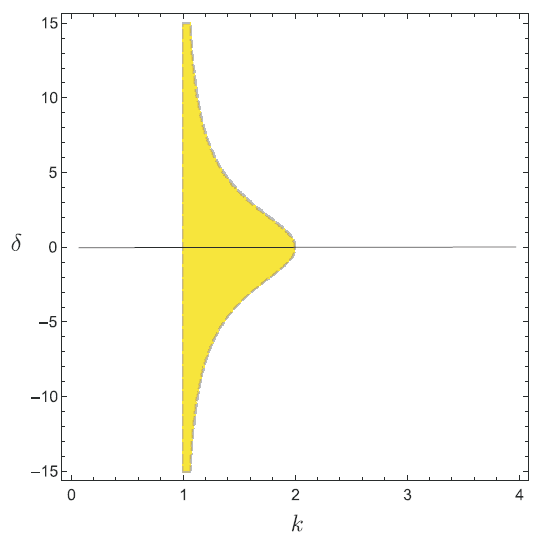

Figure 7. $D_{1}\left(k, \frac{1}{k}, \delta\right)$ is nonempty iff $(k, \delta)$ is in the shaded region.

In Figs. 6 and $7 D_{0}\left(k, \frac{1}{k}, \delta\right) \neq \varnothing$ and $D_{1}\left(k, \frac{1}{k}, \delta\right) \neq \varnothing$ iff $(k, \delta)$ is in the respective shaded region. In Fig. 8 the four first boundary curves $\Omega_{j}\left(k, \frac{1}{k}, \delta\right)$ of the regions in the $(k, \delta)$-plane, which guarantee that the regions $D_{j}\left(k, \frac{1}{k}, \delta\right) \neq$ $\varnothing(j=0,1,2,3)$ are depicted. If $k=1.08, l=\frac{1}{k}$ and $\delta=13.88$, the only two "good" regions $D_{2}(k, l, \delta)$ and $D_{3}(k, l, \delta)$ are nonempty, see Fig. 9. 


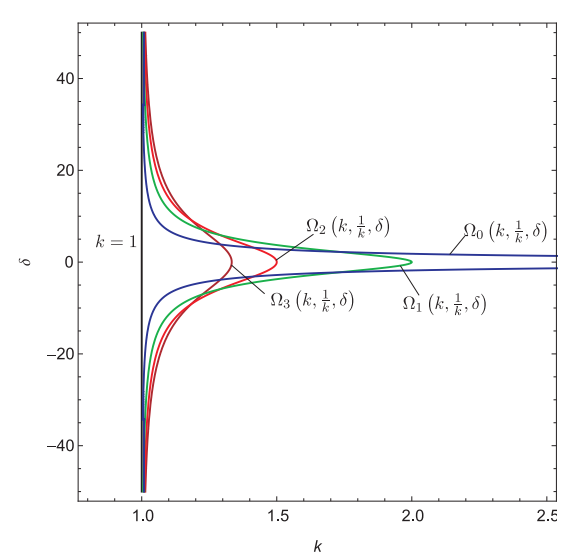

Figure 8. Boundary curves $\Omega_{j}\left(k, \frac{1}{k}, \delta\right)$ of the regions which guarantee that the regions $D_{j}\left(k, \frac{1}{k}, \delta\right)$ are nonempty $(j=0,1,2,3)$.

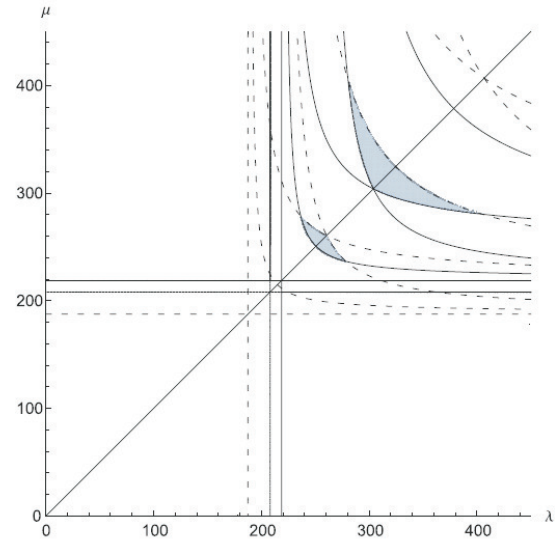

Figure 9. Only two "good" regions $D_{2}\left(k, \frac{1}{k}, \delta\right)$ and $D_{3}\left(k, \frac{1}{k}, \delta\right)$ are nonempty if $k=1.08$ and $\delta=13.88$.

Proposition 4. Suppose $0<l<k$.

1. $D_{j}(k, l, \delta)$ are open bounded connected sets for any $j=0,1,2, \ldots$;

2. $D_{j_{1}}(k, l, \delta) \cap D_{j_{2}}(k, l, \delta)=\varnothing$, where $j_{1}, j_{2} \in\{0,1,2, \ldots\}$, if $j_{1} \neq j_{2}$;

3. There are $k, l$ and $\delta$ with no "good" regions (necessarily $\delta \neq 0$ ).

4. There are $k, l$ and $\delta$ with exactly one "good" region $D_{j}(k, l, \delta)(j>0)$ (necessarily $\delta \neq 0$ ).

5. Suppose $k>1$ and $l=\frac{1}{k}$. For any $k$ and $\delta$ either no "good" regions or there exist $j, s \in\{0,1,2, \ldots\}$, where $s \geq j$, such that only nonempty "good" regions are

$$
D_{j}\left(k, \frac{1}{k}, \delta\right), D_{j+1}\left(k, \frac{1}{k}, \delta\right), \ldots, D_{j+s}\left(k, \frac{1}{k}, \delta\right) .
$$

6. If $\delta=0$, then for any $k$ there exists $s \geq 0$ such that only nonempty "good" regions are

$$
D_{0}(k, l, 0), D_{1}(k, l, 0), \ldots, D_{s}(k, l, 0) .
$$

Proof is done by elementary geometrical considerations.

\section{Conclusions}

- The introduction of a damping term in equation (2.1) does not change the spectrum essentially.

- If $f$ and $g$ are sector-bounded nonlinear functions, it is possible to define the triples $(\delta, \lambda, \mu)$ such that the problem (1.8) is solvable. 
- These sets $D_{j}(k, l, \delta)$ can be described very precisely using the properties of Fučík type spectra of auxiliary problems.

- Somewhat peculiar properties of the sets $D_{j}(k, l, \delta)$ are described in Proposition 4.

\section{Acknowledgements}

The authors would like to thank referees for valuable suggestions and useful comments.

\section{References}

[1] M.A. Aizerman. On a problem concerning the stability "in the large" of dynamical systems. Uspehi Matem. Nauk (N.S.), 4(32):187-188, 1949.

[2] S. Fučík and A. Kufner. Nonlinear Differential Equations. Elsevier, 1980.

[3] A. Gritsans and F. Sadyrbaev. Nonlinear spectra for parameter dependent ordinary differential equations. Nonlinear Anal. Model. Control, 12(2):253-267, 2007.

[4] A. Gritsans and F. Sadyrbaev. On nonlinear Fučík type spectra. Math. Model. Anal., 13(2):203-210, 2008.

http://dx.doi.org/10.3846/1392-6292.2008.13.203-210.

[5] A. Gritsans and F. Sadyrbaev. Two-parametric nonlinear eigenvalue problems. In L. Hatvani and T. Krisztin(Eds.), Proc. of the 8'th Colloquium on the Qualitative Theory of Differential Equations, Szeged, Hungary, 2007, pp. 1-14, 2008.

[6] A. Gritsans and F. Sadyrbaev. Nonlinear spectra: the Neumann problem. Math. Model. Anal., 14(1):33-42, 2009.

http://dx.doi.org/10.3846/1392-6292.2009.14.33-42.

[7] A. Gritsans and F. Sadyrbaev. Two-parameter nonlinear oscillations: the Neumann problem. Math. Model. Anal., 16(1):23-38, 2011. http://dx.doi.org/10.3846/13926292.2011.559449.

[8] A. Gritsans and F. Sadyrbaev. Nonlinear problems with asymmetric principal part. Math. Model. Anal., 17(2):217-226, 2012. http://dx.doi.org/10.3846/13926292.2012.661697.

[9] K.H. Khalil. Nonlinear Systems. Prentice Hall, 2002.

[10] M. Krasnoselskii, A. Perov, A. Povolockii and P. Zabreiko. Plane Vector Fields. GIFML, 1963. (in Russian)

[11] A.C. Lazer and P.J. McKenna. Existence, uniqueness, and stability of oscillations in differential equations with asymmetric nonlinearities. Trans. Amer. Math. Soc., 315(2):721-739, 1989.

http://dx.doi.org/10.1090/S0002-9947-1989-0979963-1.

[12] A.C. Lazer and P.J. McKenna. Large-amplitude periodic oscillations in suspension bridges: some new connections with nonlinear analysis. SIAM Rev., 32(4):537-578, 1990. http://dx.doi.org/10.1137/1032120.

[13] F. Sadyrbaev. Multiplicity in parameter-dependent problems for ordinary differential equations. Math. Model. Anal., 14(4):503-514, 2009.

http://dx.doi.org/10.3846/1392-6292.2009.14.503-514. 